surveillance. These. The status of the data sources with respect to these data elements was recorded and compared to reporting standards of the ILO, OSHA and ICD10.

Results 8 data sources were included. 4 from the Hamad Medical Corporation [HMC], the national health service provider: trauma registry, ambulance service, emergency department [3 hospitals] and mortuary. Other data sources were: Qatar Red Crescent Clinics, Public Works Authority, the Ministry of Labour and Ministry of Interior. The following data elements were collected by all sources: age, sex and nationality. External causes of injury, circumstances and nature of injury were collected by 6 or more sources, occupation by 4 sources, while the documentation of work-relatedness, and injury severity score calculation was only done by 1 , the HMC Trauma registry.

Conclusions Data collection for the estimation of WRIs in Qatar is not systematic and thus important aspects of injury prevention and safety promotion are overlooked. A mechanism to generate data in compliance with international standards is needed. In the interim, linking these data sources, through ongoing multi-sectoral collaboration will improve the quality of WRI data and inform occupational injury prevention efforts.

\section{QUAD-RELATED FATAL INJURIES 2007-2012: A COMPARISON BETWEEN AUSTRALIA AND NEW ZEALAND}

${ }^{1}$ Rebbecca Lilley, ${ }^{2}$ Tony Lower, ${ }^{1}$ Gabrielle Davie, 'Sue Wilson. 'University of Otago, New Zealand; ${ }^{2}$ University of Sydney, Australia

\subsection{6/injuryprev-2016-042156.771}

Background Serious concerns have been raised over the safety of quad bikes in Australia and New Zealand (NZ). Previous studies have sought to describe quad fatalities within each country. Given the economic similarities between Australia and NZ, cross-country comparisons could untease geographical and policy influences to inform injury prevention initiatives in both countries. This presentation describes and compares the personal, injury and environmental factors associated with quad fatalities for the period 2007-2012 using Coronial case files for Australia and NZ.

Methods Coronial case files for the period 2007 to 2012 held by the National Coronial Information System, Australia were used to identify fatalities involving a quad for both Australia and NZ using key word and mechanism searches. Information on the decedent was extracted from coronial case files. The circumstances surrounding the fatal incident was coded using the Australian Farm Injury Optimal Dataset coding framework. Comparison of the distribution of decedent, injury characteristics and event circumstances were undertaken.

Results A total of 101 quad-related fatalities were identified: 69 in Australia and 32 in NZ. The pattern of decedent characteristics was similar between both countries. Month of incident differed with $63 \%$ of Australian fatalities occurring January-June, while $66 \%$ of NZ fatalities occurred July-December. Differences where observed for primary mechanism of injury with Australian fatalities mainly involving collisions with stationary objects while NZ fatalities mainly involved quad roll-over. The presence of a slope was more commonly observed in NZ quad-related fatalities.

Conclusions Given patterns of quad-related fatalities are similar between Australia and NZ consolidation of injury prevention initiatives, particularly around quad manufacturing standards and controls, could be undertaken to develop effective prevention interventions to address commonly held quad safety concerns.

\section{GAP ANALYSIS ON PESTICIDES CONTROL AND OCCUPATIONAL HEALTH LEGISLATION IN THE UNITED ARAB EMIRATES}

${ }^{1}$ Hamda Al Dhaheri, ${ }^{1} T o m$ Loney, ${ }^{1,2}$ Tar-Ching Aw, ${ }^{3}$ Abdu Adem, 1,4Balazs Adam, ${ }^{1}$ Anza Elias, ${ }^{1}$ Michal Grivna, ${ }^{1}$ lain Blair. 'Institute of Public Health, College of Medicine and Health Sciences (CMHS), United Arab Emirates University (UAEU), Al Ain, Abu Dhabi, UAE; ${ }^{2}$ PAPRSB Institute of Health Sciences, Universiti Brunei Darussalam, Brunei Darussalam; ${ }^{3}$ Department of Pharmacology and Therapeutics, CMHS, UAEU, UAE; ${ }^{4}$ Department of Preventive Medicine, Faculty of Public Health, University of Debrecen, Debrecen, Hungary

\subsection{6/injuryprev-2016-042156.772}

Background The United Arab Emirates (UAE) is one of the leading agricultural producers in the Middle East, particularly for date fruit cultivation. Federal and local agencies are responsible for governing occupational health and safety regulations across the seven emirates. A gap analysis was performed on specific UAE legislation related to the safe use of pesticides compared to a compliance checklist developed from international best practice. Interviews with municipalities, authorities, and industry representatives were used to collect the data required in the checklist.

Description of the problem The UAE Ministry of Environment and Water reported that the average amount of pesticides used in the UAE in 2013 was five to ten times higher than in India, Europe, and the United States. Sixty-percent of UAE expatriate workers ( $\sim 5.5$ million) are unskilled migrants from less-developed countries with low education and literacy rates (39\% educated only to primary level or less). Agricultural workers lack any job-specific training and may not understand instructions on handling chemicals or pesticides safely which places them at a high risk of occupational exposure.

Results The UAE Ministry of Labour only stipulates broad federal occupational health regulations for worker protection and residency visa screening obligations for employers. There are no specific articles related to the control of hazardous chemicals such as pesticides. At the local level, only Abu Dhabi and Dubai emirates provide guidelines related to occupational specific chemical exposures; however, there is currently limited evidence of application and enforcement.

Conclusions Currently, there is inter-emirate fragmentation in the scope and enforcement of regulations related to the use and control of chemicals such as pesticides. Future legislation should include job-specific training, implementation of effective chemical hazard control methods, and health surveillance of workers exposed to pesticides.

\section{Client and Patient Safety}

Post Tue 2.18

\section{INDIAN EXPERIENCE WITH PATIENT SAFETY IN PUBLIC HEALTH}

${ }^{1}$ RK Srivastava, ${ }^{2}$ Anil Kumar. ${ }^{1}$ Former Director General Director General of Health Services, Ministry of Health and Family Welfare, Govt. of India; ${ }^{2}$ Chief Medical Officer, DGHS, Gol

10.1136/injuryprev-2016-042156.773 
Background Safety of patients in tertiary care hospitals in India was found to be a big problem. $62.9 \%$ of unsafe injections are administered every year ${ }^{1}$ in India. Estimated risk of HCAI is up to 20 times higher than industrialised countries and is approx $10 \%$ and $15-30 \%$ in acute care. $77 \%$ of all reported cases of counterfeit and substandard drugs are from developing countries. Methods A longitudinal study on patient safety practice in 12 (6 public +6 private) multi-specialty hospitals* was conducted by DGHS from 2005-2011 with objective of identifying, analysing and improving the gaps in hospital patient safety system, so that it is compliant to WHO guidelines on patient safety 2005. The study took up safety practice for hand hygiene, surgery, injection, medication, blood transfusion, infection control and hospital waste management as core areas of hospital patient safety system. Data were collected \& analysed annually and at the end of study. It provided for annual gap identification and introduction of corrective measure in each core area and also for system development at end of study in areas of advocacy, infrastructure, capacity building and funding for practice of patient safety in hospitals.

Results This study created a patient safety unit in DGHS, a patient safety committee in the hospitals, a targeted advocacy programme, guidelines for survey and root cause analysis, protocols for safety practice in hospitals, training manuals, standard reporting format for adverse events, supportive monitoring system , and annual and study- end evaluation of outcome in terms of mortality and adverse events. Policy and practice changes like introduction of auto-disable syringe in public health (2009), revision of guidelines for disposal and syringe/needle under bio-medical disposal rules (2010), introduction of antibiotic policy in tertiary hospitals, strengthening of pharmaco-vigilance programme took place during this period. Success stories, reports of hospital, study report and technical report were documented.

Conclusions The study was able to showcase relevance of patient safety system in tertiary hospitals for better quality outcome.

*Safdarjang Hospital, Ram Manohar Lal Hospital, AIIMS, Kalawati Saran Hospital, G. B. Pant Hospital, and GTB Hospital in public Health Sector - 6 National Accreditation Board for Hospital and Healthcare Providers approved private hospitals of Max group

MoHFW - Ministry of Health \& Family Welfare

HCAI - Hospital Care Associated Infections

- (CLEN Program Evaluation Network (IPEN) for Department of Family Welfare, MoHFW)

\section{FACTORS THAT IMPROVE THE SAFETY OF PATIENT CARE AS EXPERIENCED BY EMERGENCY MEDICAL CARE PROVIDERS}

${ }^{1}$ Mari Salminen-Tuomaala, ${ }^{2}$ Päivi Leikkola, ${ }^{3}$ Riitta Mikkola, ${ }^{4}$ Eija Paavilainen. ${ }^{1}$ Seinäjoki University of Applied Sciences, School of Health Care and Social Work, Finland; '²einäjoki Central Hospital, Finland; ${ }^{3}$ University of Tampere, School of Health Sciences, Finland; ${ }^{4}$ University of Tampere, School of Health Sciences, South Ostrobothnia Hospital District

\subsection{6/injuryprev-2016-042156.774}

Background An increasing number of acutely ill patients are not transported to hospital but treated in their homes or another location. Special attention should be paid to factors that affect patient safety (Ministry of Social Affairs and Health 2014.)

Methods The study describes factors that promote the safety of patient care in out-of-hospital emergency medical care, as experi- enced by care providers. The data were collected by semi-structured interviews $(\mathrm{n}=15)$ with care providers selected by discretionary sampling. The material was analysed using inductive content analysis.

Results Patient safety improves with successful consultation with the emergency physician and with holistic consideration of all factors that affect the patient's coping. Safety is further promoted by the emergency care provider's theoretical, practical and attitudinal competencies and co-operation skills. Situational sensitivity is an important factor intertwined with the care provider's personality, emotional intelligence and discretion. Moreover, safety can be improved by making certain that individual procedures are carried out safely and by ensuring continuity of care and coping with home care. Finally, management practices that aim at workers' wellbeing at work increase patient safety.

Conclusions A study of factors that improve or impair patient safety can help care providers anticipate threats and prevent risks.

\section{PATIENT SAFETY IN THE OPERATING ROOM: NURSES' EXPERIENCE AND ATTITUDES IN CYPRUS}

${ }^{1}$ Petya Trendafilova, ${ }^{2}$ Elisabeth Michael. ${ }^{1}$ Medical University - Sofia, Faculty of Public Health, Sofia, Bulgaria; ${ }^{2}$ Makarios III Hospital, Nicosia, Cyprus

\subsection{6/injuryprev-2016-042156.775}

Background Effective communication skills in the operating room (OR) promote team behaviour and usually result in good patient outcomes. Communication and collaboration constitutes an integral role during this process both with patients and with inter-professionals when working in such a highly specialised setting as an OR. Patient and staff safety in the OR has never been investigated in Cyprus before.

Methods One of the most often used tools to assess safety culture in an organisation is the Safety Attitudes Questionnaire (SAQ) (The Evidence Centre, 2011 \& Sexton et al, 2006). SAQ is a psychometric instrument used for evaluating healthcare providers and especially operating nurses' attitudes regarding patient safety and climate. Six major factors are measured through the use of modified SAQ: teamwork climate, job satisfaction, management perception, safety climate, working conditions and stress recognition. A random sample of 112 OR nurses was studied from Makarios III Hospital which is one of the two major public hospitals in Nicosia.

Results The sample was comprised of 30 males and 82 females: the majority was working as permanent staff in the hospital and their experience varied from $0-25$ years both in total as well as in the specific hospital. Overall participants believed that issues related to guidelines, teamwork and patient safety need further improvements. Males have significantly higher scores regarding experience with safety issues in the OR compared to their female counterparts. Interestingly, the lowest scores regarding nurses' notions and opinions regarding OR safety were expressed by the most experienced participants, who had more than 25 years of work experience. This is an indication of the need for further improvements in the in-service training which currently takes place in the Cyprus health system.

Conclusions More experienced staff might be in need for education regarding more recent safety procedures and techniques followed in the OR. 